Vol. 13, n 1 | 2009

Varia

\title{
Bronislaw Geremek (1932-2008)
}

\section{Elżbieta Kaczyńska}

Traducteur : Claude Gauthier

\section{(2) OpenEdition}

\section{Journals}

Édition électronique

URL : https://journals.openedition.org/chs/727

DOI : $10.4000 /$ chs. 727

ISSN : 1663-4837

Éditeur

Librairie Droz

Édition imprimée

Date de publication : 1 mars 2009

Pagination : 165-167

ISBN : 978-2-600-01295-9

ISSN : 1422-0857

\section{Référence électronique}

Elżbieta Kaczyńska, "Bronislaw Geremek (1932-2008) », Crime, Histoire \& Sociétés / Crime, History \&

Societies [En ligne], Vol. 13, n¹ | 2009, mis en ligne le 25 mars 2009, consulté le 25 mars 2022. URL http://journals.openedition.org/chs/727 ; DOl : https://doi.org/10.4000/chs.727 


\section{Nécrologie}

\section{BRONISLAW GEREMEK (1932-2008)}

Le 13 juillet 2008 est mort tragiquement dans un accident de voiture, l'historien et homme d'État, l'un des Polonais les plus célèbres, le Professeur Bronislaw Geremek.

Son activité politique en Pologne et sur la scène internationale ne peut être dissociée de l'étude qu'il a menée sur l'histoire à propos de laquelle il a écrit. Il a étudié plusieurs siècles d'histoire pour transférer son intérêt à l'époque moderne comme en témoigne son dernier livre ${ }^{1}$. Aujourd'hui il aide à mieux comprendre les particularités du passé, passé qui influence les éléments de notre vie contemporaine. Geremek était interessé par la continuité et le changement, pour reprendre les termes d'un ami et maître, Fernand Braudel. Geremek était particulièrement sensible aux problèmes sociaux tels que la pauvreté, la place des groupes marginaux dans la société, la tolérance, l'exclusion de ceux qui vivent en périphérie de l'«establishment » d'hier et d'aujourd'hui. Il affirme, lors du discours d'inauguration au Collège de France en 1993 que l'exclusion sociale non seulement existait au Moyen-Âge mais elle est bien présente dans toutes les sociétés, même si elle change de forme et de sujet ${ }^{2}$. Cet intérêt était déjà manifeste dans le premier livre publié ${ }^{3}$.

Bronislaw Geremek a terminé ses études d'histoire à l'Université de Varsovie en 1954. Il a œuvré dans l'équipe d'études médiévales de Marian Malowist. En 1960 , il a obtenu son doctorat puis en 1972 son doctorat d'État. Cette longue période d'attente à la titularisation s'explique par son profond engagement dans l'opposition politique polonaise et par les années d'incarcération durant la loi martiale. Il a complété ses études nationales au Smithsonian Institute aux États-Unis ainsi qu’à l'École Pratique des Hautes Études en France. De la fin de ses études jusqu'à la loi martiale, il a été employé par l'Institut d'Histoire de l'Académie des Sciences de Pologne sauf pendant les années 1960-1965 où il dirigea le Centre de la Culture

Geremek, B., Litość i szubienica. Dzieje nędzy i miłosierdzia (L'histoire de la pauvreté et la charité), Varsovie, Czytelnik, 1989.

2 Geremek, B., Histoire sociale : exclusions et solidarité. Leçon inaugurale faite le vendredi 8 janvier 1993, Paris Collège de France.

3 Geremek, B., Życie codzienne w Paryżu Franciszka Villona (La vie quotidienne à Paris de François Villon), Varsovie, 1972 (et aussi: Le monde de François Villon, Rome ed. Laterza, 1990). Le commerce de Novgorod l'Occident au Moyen-Âge, Paris, Armand Colin, 1964. Conjointement écrit avec Kazimierz Piesowicz: Ludzie, towary, pienqdze (Les gens, les biens, l'argent), Varsovie, Wiedza Powszechna, 1968. 
Polonaise à Paris. De nombreuses traductions de ses livres, 23 doctorats honorifiques, sa nomination comme professeur invité en 1992 au Collège de France témoignent de sa reconnaissance non seulement en Pologne mais à l'étranger. Il a été également membre de nombreuses associations, y compris le Pen Club.

Geremek aimait citer les mots d'Éric Hobsbawm «qu'il n'y a pas d'autre histoire que sociale car dans la société il y a de constantes interactions entre l'individuel et le collectif et entre la vie matérielle et la psychologie collective». Il dit aussi que «la notion d'histoire sociale, en proclamant l'étude de l'homme dans l'environnement social, se réfère à l'histoire tout court, annonce une approche totalisante du fait humain». Telle est la réalité de la vie humaine sur une base quotidienne, et pas seulement pour les riches et éclairés, mais aussi pour les gens du peuple, les mendiants et les criminels. L'histoire raconte l'homme dans son environnement, a annoncé «une approche totalisante de l'homme». Le succès de l'historiographie internationale contemporaine «l'union entre l'érudition rigoureuse, une problématique nouvelle et un regard anthropologique» a de toute évidence attiré Geremek vers les recherches historiques ${ }^{4}$.

En 1990 eut lieu, à l'ambassade de France à Varsovie, un passionnant dialogue entre deux célébrités: Geremek et le Français Georges Duby. Ainsi est né le livre Passions communes sous la direction de Philippe Sainteny ${ }^{5}$. Les idées émises par les deux chercheurs est le credo en termes d'approche à leur métier, à la méthodologie, à la problématique et même comme mission pour un historien. Les Passions communes donnent envie de relire l'œuvre de ces auteurs notamment au sujet de la plèbe des villes, des mendiants, des vagabonds, des exclus sociaux, sur la répression et sur l'exclusion sociale et en contrepartie sur la charité. Geremek définit la marginalisation ainsi: une vie en marge de la société qui appelle une participation limitée comme la vie des moines et celle des femmes. En revanche, l'exclusion érige des barrières, un mur infranchissable au delà duquel se placent ceux qui refusent de se subordonner aux valeurs convenues ou qui ne sont pas acceptés par la société. Ceci n'est pas l'équivalent de la pauvreté; un pauvre «n'est pas inutile au monde», il joue un rôle dans la société, même les fous et les lépreux sont les intermédiaires entre Dieu et les pécheurs. Mais «un bon pauvre» doit se soumettre à son destin sinon il se place dans la catégorie des exclus et des «inutiles au monde». Geremek affirme que la charité envers les pauvres est inhérente au Moyen-Âge et qu'elle commence à décliner $\mathrm{au} \mathrm{XVI}^{\mathrm{e}}$ et surtout au $\mathrm{XVII}^{\mathrm{e}}$ siècle où l'on observe une tendance à les enfermer dans les asiles et hôpitaux spécialisés ${ }^{6}$. L'intérêt de Geremek pour la plèbe et les marginaux couvre aussi la criminalité et la répression, toutefois sa recherche vise la vie réelle et non pas l'histoire du droit ou des lois ${ }^{7}$. Il s'agirait ici de parler non pas de criminalité mais plutôt des criminels dans leur environnement social. Il fait des liens entre les relations des communautés urbaines et les vagabonds, la prostitution, la fornication, et dresse des frontières fluides entre le commerce itinérant et les groupes criminels organisés. Il remet en cause le mouvement social faussement attribué aux marginaux.

\footnotetext{
4 Geremek, B., Histoire sociale, passim.

5 Duby, G., Geremek, B., Passions communes, Varsovie, PWN, 1995.

6 Le Stripe di Caino: l'immagine dei vagabondi dei poveri nelle letterature europee dal XV al XVII secolo, Milano, Il Saggiatore, 1988.

7 Geremek, B., Les gens en marge du Paris médiéval (XIV ${ }^{\mathrm{e}}-\mathrm{XV}^{\mathrm{e}}$ siècles, $2^{\mathrm{e}}$ édition (1 ${ }^{\mathrm{e}}$ édition 1972), Poznan, Société des Amis des sciences de Poznan, 2003.
} 
Pour Geremek le paysage de la vie urbaine a été enrichi par ce qui est son quotidien, bien que généralement peu reluisant: émeutes et bagarres, vol, abandon d'enfant, mendicité, exécutions, fornication. Dans un chapitre intitulé «topographie sociale», elle s'avère possible si l'on tient compte des problématiques et paramètres mentionnés supra, souvent ignorés par d'autres historiens. La source des documents a été puisée dans les archives municipales, les anecdotes, le répertoire de théâtre, la littérature de gangster et les œuvres littéraires.

Bronislaw Geremek est mieux connu dans le monde en tant qu'homme d'État, pourtant c'est aussi un grand historien. En qualité de politicien comme d'historien, il a eu le courage de ses convictions et celui de chercher ce que d'autres refusaient de faire ou ne savaient pas faire.

Elżbieta Kaczyńska Professeur à l'Université de Varsovie (traduction Claude Gauthier)

\section{DAVID JOHN PHILIPS (1946-2008)}

It is with deep sadness that we announce the death of our colleague David Philips. He died of a heart attack while on vacation in Queensland last summer. To a good number of us in the world of criminal justice history he was both colleague and friend.

David was born in South Africa in 1946, the son of a well known barrister and judge. He was educated at the University of Witwatersrand and at Magdalen College, Oxford, where he took undergraduate degrees in history. He completed a D.Phil degree at Nuffield College, Oxford.

Before attending university he served in the South African navy, and once described to this writer his feelings as his ship cruised by Robben Island where Nelson Mandela was incarcerated. Because of his detestation of the apartheid regime, he did not return home after completing his doctorate, but emigrated to Australia where he spent a long and productive career teaching at The University of Melbourne.

Although he taught history, he had a life-long attraction to the law. In his later years, while still teaching in the history department, he completed a law degree at Melbourne, took a leave from his academic position and briefly practiced in a local law firm. His return to the history department was marked by a turn to the exploration of recent South African history, producing a number of essays on the South African Truth and Reconciliation Committee. He also researched and wrote extensively on indigenous land rights in Australia and other parts of the British Empire.

The bulk of his academic career, however, was devoted to the field of criminal justice history. He produced numerous articles, a seminal book, Crime and Authority in Victorian England (London, 1977), and co-authored (with Robert D. Storch) Policing Provincial England 1829-1856: The Politics of Reform (London and New York, 1999). He also published a fascinating biography of one of the little known moral entrepreneurs of nineteenth-century England, William Augustus Miles, a supposed royal bastard who conducted interviews for Edwin Chadwick and who became the chief of police in colonial Sydney, William Augustus Miles: Crime Policing and Moral Entrepreneurship in England and Australia (Melbourne, 2001). 